\title{
Using qualitative and mixed methodologies to explore hierarchy in the operating room
}

\author{
Anne Wong, MD, PhD
}

Received: 23 February 2015/Accepted: 13 March 2015/Published online: 19 March 2015

(C) Canadian Anesthesiologists' Society 2015

In this issue of the Journal, Bould et al. ${ }^{1}$ report on the findings of an intriguing mixed methods study that investigates the phenomenon of hierarchy in the operating room setting and its effect on decision-making. In this study, a simulated intraoperative crisis scenario was created in which resident participants were ordered by a faculty anesthesiologist (whom they had not previously met) to transfuse a Jehovah's Witness patient contrary to the patient's explicit written order. This scenario took place in either a "high" or a "low hierarchy" operating room environment where scripted interactions between team members were either formal and impersonal or informal and friendly. The simulations were video recorded, and the behaviours of the residents in challenging the order to transfuse were rated using the modified Advocacy Inquiry Scale (mAIS). ${ }^{2}$ The results of this quantitative component of their study had been previously reported. ${ }^{2}$

This present paper focuses on the findings from the qualitative part of their study where the residents were subsequently debriefed and interviewed post-simulation. As qualitative and mixed methods studies are relatively less common in anesthesia research, it is useful to review these approaches prior to commenting further on the findings of this study.

Healthcare research has been dominated traditionally by quantitative research; recently, however, qualitative research has become increasingly used. ${ }^{3-5}$ Of the various qualitative methodologies, the grounded theory methodology has gained the most popularity in healthcare

\footnotetext{
A. Wong, MD, PhD ( $₫)$

Department of Anesthesia, McMaster University, 1280 Main St. W. HSC 2V9 or MDCL 3523, Hamilton, ON L8S 4K1, Canada e-mail: wongan@mcmaster.ca
}

research, due in part to its focus on generating theory from the empirical data and its specified methodological procedures. $^{3-5}$ While both quantitative and qualitative research aim to represent phenomena accurately, they approach this task from different epistemological frameworks, purposes, and methodological procedures. ${ }^{6,7}$

Quantitative research is based on a "positivist" paradigm or worldview that holds that there is a true objective reality that can be discovered through rigorous scientific inquiry in settings that are controlled to prevent interference from biases. ${ }^{4,6,7}$ The postpositivist paradigm modifies this stance in accepting that reality can only be approximated due to the inherent limitations of scientific inquiry. ${ }^{4}$ Using a hypothetico-deductive approach, quantitative research seeks to test, predict, or verify hypotheses. Sampling is random and sample size is determined in order to generalize to the population. Data are typically numeric. The rigour of quantitative research is evaluated on standardized criteria such as reliability, validity, and replicability. $^{7}$

Qualitative research is generally premised on a "constructivist" paradigm that holds that, rather than being a singular entity, reality is contextual and constructed through engagement of the investigator with the phenomena. ${ }^{4,6,7}$ Therefore, there may be more than one valid interpretation or representation of reality. Rather than trying to control for bias, the investigator reflexively acknowledges how his or her perspectives and experiences influence the research process and its interpretation. Rather than testing hypotheses, qualitative research is inductive, aiming to describe, understand, and explain social phenomena in their natural contexts. Data are nonnumeric, typically with words and phrases from interviews and observations, but they could also include documents, artefacts, and visual material. ${ }^{6}$ Sampling is 
purposeful rather than random, aimed at selecting the most appropriate cases to answer the research questions. Final sample size is determined when analysis reaches "saturation", i.e., the point at which further analysis of incoming data no longer produces any new insights or findings to the emergent theory. $3,4,6,8$

Qualitative research has been critiqued as being too subjective, non-generalizable, and lacking in transparency and rigour. $^{7}$ It has been accepted that quantitative and qualitative research should have different criteria for rigour given their different paradigms, purposes, and methodologies, but the actual criteria have been vigorously debated. ${ }^{6,7}$ For example, Lincoln and Guba proposed that trustworthiness, characterized by "credibility", "transferability", "dependability", and confirmability, should be the main criterion for judging qualitative research, whereas others argue that the heterogeneity of qualitative methodologies warrants criteria specific for each methodology. ${ }^{7}$

Grounded theory is a qualitative methodology originated by sociologists Barney Glaser and Anselm Strauss in 1967. ${ }^{49}$ Glaser and Strauss considered it important to generate theory from empirical data, "grounded" data, rather than to test theories using empirical studies. In its original form, grounded theory developed from positivist and postpositivist assumptions that, when properly conducted, the true nature of the phenomena would emerge from the data. ${ }^{4,5}$

Since that seminal publication, grounded theory has evolved into a number of different approaches or "schools". ${ }^{3-5}$ Despite the different schools, there are a number of common elements that define grounded theory methodology. These include: an inductive approach to the data with an initial broad and open coding of words and phrases with descriptors; purposeful theoretical sampling; iterative data gathering and analysis wherein data are analyzed shortly after collection; constant comparison of current and prior data; secondary or axial coding that merges codes under larger conceptual categories (which generates memos in order to develop an evolving understanding of the relationships amongst the concepts); and production of a substantive theory from the culmination of this process. ${ }^{3-5,8}$ Kennedy and Lingard ${ }^{8}$ distinguish between grounded theory research, which uses all the aforementioned elements, and studies that use the grounded theory approach to analysis. Bould et al.'s study ${ }^{1}$ would fall into the latter category, as the qualitative phase is part of a mixed methods study, and sampling was neither iterative nor purposeful.

In grounded theory methodology, Charmaz ${ }^{5}$ explicates four criteria for assessing rigour: resonance, credibility, originality, and usefulness. Resonance refers to the degree to which the findings make sense and fully capture the phenomena. Credibility refers to the methodological fit of research design and the degree to which the research procedures are followed and justifiably lead to the final interpretation and theory. Originality refers to the degree to which the theory offers new insights and advances understanding. Usefulness refers to the applicability of the theory.

In the early 1990s, mixed methods research (combining both quantitative and qualitative methods) became formalized as a distinct research methodology. ${ }^{10}$ Because of the paradigmatic differences between quantitative and qualitative methodologies, some have argued that the two cannot be coherently integrated. ${ }^{10}$ On the other hand, others argue that mixed methods offer opportunities to combine the strengths of both methodologies to gain a fulsome understanding of the phenomena. ${ }^{10}$

Sydor et al. have engaged in a unique investigation that uses a simulated environment in order to create the relatively new concept of "sociological fidelity" with respect to hierarchy amongst operating room team members. ${ }^{2}$ In this simulation, purportedly meant to study team behaviour, the investigators used deliberate deception in order to examine the response of residents to directions from a faculty member to transfuse a Jehovah's Witness patient contrary to explicit written instructions from the patient. The deception involved telling the residents that this faculty member was a recent appointment to their department, although, unbeknownst to them, the person was actually an actor and part of the research team. As an aside, the use of deliberate deception as part of "sociological fidelity" raises interesting ethical issues that are beyond discussion in this editorial; however, it would be important to examine these matters in the future.

Interestingly, despite being aware of the patient's refusal for blood transfusion, the majority of residents went ahead and gave the blood irrespective of being in the high or the low hierarchical environment. The mAIS scores did not differ between the two groups. ${ }^{2}$ This finding suggests that there are deeper structures of hierarchy that act to constrain the residents' behaviour. This finding has implications for decision-making and patient safety in crisis situations.

The residents were subsequently debriefed post simulation to reveal the deception. For a further investigation of their perceptions and actions during the simulation, individual interviews were subsequently conducted. The interviews were recorded and transcribed and then analyzed in order to generate a theory of hierarchy and its effect on decision-making in the operating room. Data from the debriefing were also selectively used in the analysis. 
The authors described in detail how they collected the data and used the grounded theory analytic approach to arrive at their findings, including an analytic diagram that illustrates how they moved from coding their data to developing their conceptual model. They found three major conceptual themes associated with the phenomena of hierarchy in the operating room: operating room culture, coping mechanisms, and effects of hierarchy. Each theme was moderated in turn by other subthemes. The authors found a strong relationship between the residents' behaviour and their perception of hierarchy in the operating room.

By integrating the findings from the quantitative and qualitative phases of their study, the authors further propose a theoretical model for challenging authority in a crisis situation. Their model is based on the individual's position within the hierarchical framework of the operating room and on the perception that such a challenge would have either a detrimental or a beneficial effect. The four quadrants of this model describe the various scenarios and conditions that may predispose an individual to take one course of action over another. The authors suggest further studies to examine the validity and utility of this model.

This study illustrates how the use of qualitative research enables the investigators to go beyond the mAIS findings to explore the underlying reasons and, furthermore, to propose a theoretical model by integrating their findings. Nevertheless, it also shows that mixed methods research can be challenging to design and carry out because of the requisite methodological expertise and considerations of rigour. These challenges can be met with further development of the field as well as interdisciplinary research collaborations.

This type of research is very important as we go beyond the issues of individual competencies in providing safe anesthetic care. Given that medical error is currently conceptualized as a systems failure, as exemplified in Reason's ${ }^{11}$ "Swiss cheese model", the examination of broad sociological processes such as hierarchy represents another important level of analysis of "latent" or "hidden conditions" in the surrounding environment that may contribute to error. The concept of sociological fidelity is innovative and provocative and has the potential for advancing the research and work in patient safety. Qualitative methodologies and mixed methods are well suited for exploring and understanding social processes and behaviours. A better understanding of these processes may contribute to a better awareness of environmental factors that can then be explicitly addressed and taught. In turn, this may help enhance effective communication, overall team functioning, and ultimately patient safety care and outcomes.

\section{Méthodologies qualitatives et mixtes pour étudier les phénomènes de hiérarchie en salle d'opération}

Dans ce numéro du Journal, Bould et coll. relatent les résultats d'une étude à méthodologie mixte intrigante qui examinait le phénomène de hiérarchie en salle d'opération et son effet sur la prise de décision. Dans cette étude, on a simulé un scénario de crise peropératoire dans lequel un patron anesthésiologiste ordonnait aux résidents participants (qui ne le connaissaient pas encore) de transfuser un patient témoin de Jéhovah, contrairement aux instructions écrites explicites du patient. Cette mise en scène a eu lieu dans un environnement de salle d'opération considéré comme étant « très hiérarchisé » ou " peu hiérarchisé » et dans lequel les interactions scénarisées des membres de l'équipe étaient formelles et impersonnelles ou informelles et amicales. Les simulations ont été enregistrées sur vidéo, et les comportements des résidents concernant la remise en question de l'ordre de transfuser ont été évalués à l'aide de l'échelle mAIS (modified Advocacy Inquiry Scale, ou «échelle modifiée d'évaluation de la défense des intérêts »). ${ }^{2}$ Les résultats de cette composante quantitative de l'étude ont déjà été publiés ailleurs. ${ }^{2}$

L'article présenté ici se concentre sur les résultats qualitatifs de leur étude, soit les résultats tirés du débriefing des résidents après la simulation. Les études qualitatives et de méthodologie mixte sont relativement peu courantes dans la recherche en anesthésie; par conséquent, il convient de passer en revue ces approches avant d'examiner davantage les résultats de cette étude en particulier.

La recherche en soins de santé a traditionnellement été dominée par la recherche quantitative; toutefois, on observe récemment une utilisation croissante de la recherche qualitative. ${ }^{3-5}$ Parmi les diverses méthodologies qualitatives, la méthodologie dite de la théorie à base empirique est désormais la plus populaire dans la recherche en soins de santé, en partie en raison de son emphase sur la génération de théorie basée sur les données empiriques et de ses processus méthodologiques spécifiés. $^{3-5} \mathrm{La}$ recherche quantitative et la recherche qualitative cherchent toutes deux à représenter les phénomènes de façon précise, mais elles abordent cet objectif à partir de différents cadres épistémologiques et avec différents buts et procédés méthodologiques. ${ }^{6,7}$

La recherche quantitative se fonde sur un paradigme ou une vision du monde «positiviste", paradigme selon lequel il existe une véritable réalité objective qui peut être découverte grâce à un examen scientifique rigoureux 
dans des environnements contrôlés de façon à prévenir toute interférence due à un biais. ${ }^{4,6,7}$ Le paradigme post-positiviste modifie cette position en acceptant qu'on puisse se rapprocher de la réalité uniquement en raison des limites inhérentes à la recherche scientifique. ${ }^{4}$ En se fondant sur une approche d'hypothèse et de déduction, la recherche quantitative cherche à tester, prédire ou vérifier des hypothèses. L'échantillonnage est aléatoire et la taille d'échantillon déterminée afin de pouvoir généraliser les résultats à la population. Les données sont fréquemment numériques. La rigueur de la recherche quantitative est évaluée selon des critères normalisés tels que la fiabilité, la validité et la reproductibilité. ${ }^{7}$

La recherche qualitative, quant à elle, se fonde en règle générale sur un paradigme «constructiviste » selon lequel, plutôt que d'être une entité singulière, la réalité est contextuelle et construite par le biais de la relation entre le chercheur et les phénomènes qu'il étudie., ${ }^{4,6,7}$ Ainsi, il peut y avoir plus d'une interprétation ou représentation valable de la réalité. Plutôt que d'essayer de contrôler le biais, le chercheur reconnaît d'office que ses perspectives et ses expériences influencent le processus de recherche et son interprétation. Plutôt que de tester des hypothèses, la recherche qualitative est inductive et a pour objectif de décrire, de comprendre et d'expliquer des phénomènes sociaux dans leurs contextes naturels. Les données sont non numériques et contiennent souvent des mots ou phrases tirés d'entretiens et d'observations, mais elles pourraient également comprendre des documents, des artéfacts et du matériel visuel. ${ }^{6}$ L'échantillonnage est ciblé plutôt qu'aléatoire et vise à choisir les cas les plus adaptés pour répondre aux questions de recherche. La taille d'échantillon finale est déterminée lorsque l'analyse atteint son point de «saturation », soit le point où toute analyse future de données rentrantes ne génère plus de nouvelles perspectives ou de nouveaux résultats pour la théorie émergente. ${ }^{3,4,6,8}$

On a critiqué la recherche qualitative en lui reprochant d'être trop subjective, non généralisable et de manquer de transparence et de rigueur. ${ }^{7}$ Il est accepté que les recherches quantitative et qualitative devraient disposer de critères différents en matière de rigueur étant donné leurs divergences en matière de paradigmes, d'objectifs et de méthodologies, mais le choix de ces critères fait l'objet de débats animés. ${ }^{6,7}$ Par exemple, Lincoln et Guba ont proposé que la fiabilité ou 'trustworthiness', caractérisée par la « crédibilité », la «transférabilité », la « constance » et la « confirmabilité », représente le critère principal d'évaluation de la recherche qualitative; d'autres pensent que l'hétérogénéité des méthodologies qualitatives justifient le recours à des critères spécifiques à chaque méthodologie. ${ }^{7}$

La théorie à base empirique est une méthodologie qualitative créée par les sociologues Barney Glaser et
Anselm Strauss en 1967. ${ }^{4,9}$ Selon Glaser et Strauss, il était important de générer des théories fondées sur des données empiriques, des données « ancrées », plutôt que de tester des théories en se basant sur des études empiriques. Dans sa forme originale, la théorie à base empirique s'est développée sur la base d'hypothèses positivistes et post-positivistes selon lesquelles, si elle était bien menée, la nature véritable des phénomènes émergerait des données. ${ }^{4,5}$

Depuis la publication de cet article phare, la théorie à base empirique a évolué en plusieurs différentes approches ou « écoles ». ${ }^{3-5}$ Malgré ces différentes écoles, il existe plusieurs éléments communs qui définissent la méthodologie de la théorie à base empirique. Parmi ceux-ci, on compte: une approche inductive des données avec un codage initial ouvert et vaste des mots et phrases avec des descripteurs; un échantillonnage théorique ciblé; une collecte et une analyse des données itératives dans lesquelles les données sont analysées peu après leur collecte; une comparaison constante des données actuelles et antérieures; un codage secondaire ou axial qui fusionne les codes en catégories de concepts plus vastes (ce qui génère des mémos permettant de mettre au point une compréhension évolutive des relations entre les concepts); et la production d'une théorie substantielle de la culmination de ce processus. ${ }^{3-5,8}$ Kennedy et Lingard ${ }^{8}$ font la distinction entre la recherche de la théorie à base empirique, qui utilise tous les éléments mentionnés ci-dessus, et les études qui utilisent l'approche de la théorie à base empirique pour leur analyse. Selon cette distinction, l'étude de Bould et coll. ${ }^{1}$ appartiendrait à la seconde catégorie, étant donné que la phase qualitative fait partie d'une étude à méthodologie mixte, et que l'échantillonnage n'était ni itératif ni ciblé.

Dans la méthodologie de la théorie à base empirique, Charmaz ${ }^{5}$ définit quatre critères qui permettent d'évaluer la rigueur d'une étude: la résonance, la crédibilité, l'originalité et l'utilité. La résonance fait référence à la mesure dans laquelle les résultats sont sensés et saisissent complètement les phénomènes. La crédibilité touche à l'adéquation de la méthodologie de recherche et à la mesure dans laquelle les processus de recherche sont respectés et mènent de façon justifiée à l'interprétation et la théorie finales. Par originalité, on entend la mesure dans laquelle la théorie propose de nouvelles perspectives et fait évoluer la compréhension. L'utilité fait référence à l'applicabilité de la théorie.

Au début des années 1990, la recherche méthodologique mixte (celle qui combine les méthodes quantitative et qualitative) s'est formalisée en une méthodologie de recherche à part. ${ }^{10}$ En raison des différences de paradigmes entre les méthodologies quantitative et qualitative, certains chercheurs étaient d'avis qu'il était impossible d'intégrer les 
deux méthodes de façon cohérente. ${ }^{10} \mathrm{D}$ 'autres pensaient au contraire qu'une méthodologie mixte offrirait des possibilités de combiner les forces des deux méthodologies afin de parvenir à une compréhension plus approfondie des phénomènes. ${ }^{10}$

Sydor et coll. ont mené une étude unique qui se sert d'un environnement simulé pour (re)créer le concept relativement récent de «fidélité sociologique » en ce qui touche à la hiérarchie parmi les membres de l'équipe de la salle d'opération. ${ }^{2}$ Dans cette simulation, dont le but spécifique était d'étudier le comportement de l'équipe, les chercheurs ont délibérément menti afin d'étudier la réaction de résidents aux ordres d'un patron, soit de transfuser un patient témoin de Jéhovah et ce, contrairement aux instructions écrites explicites du patient. La tromperie consistait à dire aux résidents que ce patron était nouveau dans leur département alors que, sans qu'ils le sachent, cette personne était en fait un acteur et faisait partie de l'équipe de recherche. Entre parenthèses, le recours à une supercherie délibérée dans le cadre d'une étude de "fidélité sociologique » soulève d'importantes questions déontologiques qui vont au-delà des objectifs de cet éditorial; toutefois, il serait important d'examiner ces questions à l'avenir.

Fait intéressant, malgré le fait qu'ils étaient conscients du refus de transfusion du patient, la plupart des résidents ont suivi les ordres du patron et ont donné du sang, indépendamment du type d'environnement dans lequel ils se trouvaient, qu'il soit très hiérarchisé ou peu hiérarchisé. Les scores mAIS étaient semblables dans les deux groupes. ${ }^{2}$ Ce résultat suggère qu'il existe des structures hiérarchiques plus profondes qui agissent pour contraindre les comportements des résidents. Ce résultat a des implications quant à la prise de décision et la sécurité des patients en situation de crise.

Après la simulation, les résidents ont été débriefés et on leur a révélé la supercherie. Afin d'étudier de manière plus approfondie leurs perceptions et leurs actions pendant la simulation, des entretiens individuels ont ensuite été réalisés. Les entretiens ont été enregistrés et transcrits, puis analysés afin de générer une théorie de la hiérarchie et de son effet sur la prise de décision en salle d'opération. Certaines données tirées des séances de débriefing ont également été utilisées dans l'analyse.

Les auteurs font une description détaillée de la façon dont ils ont colligé les données et utilisé l'approche analytique de la théorie à base empirique afin de parvenir à leurs résultats, notamment un diagramme analytique qui illustre la façon dont ils sont passés du codage de leurs données à la mise au point de leur modèle conceptuel. Ils ont découvert trois thèmes conceptuels majeurs associés aux phénomènes de hiérarchie en salle d'opération: la culture de la salle d'opération, les mécanismes de survie, et les effets de la hiérarchie. Chaque thème était ensuite modéré par d'autres sous-thèmes. Les auteurs ont observé une relation forte entre le comportement des résidents et leur perception de la hiérarchie en salle d'opération.

En intégrant les résultats des phases quantitative et qualitative de leur étude, les auteurs proposent en outre un modèle théorique pour remettre l'autorité en question dans une situation de crise. Leur modèle se fonde sur la position de l'individu dans le cadre hiérarchique de la salle d'opération et sur la perception qu'une telle remise en question aurait un effet soit préjudiciable, soit bénéfique. Les quatre quadrants de ce modèle décrivent les divers scénarios et conditions qui pourraient prédisposer un individu à choisir une ligne de conduite plutôt qu'une autre. Les auteurs suggèrent que d'autres études soient réalisées afin de mettre à l'épreuve la validité et l'utilité de ce modèle.

Cette étude illustre comment le recours à une méthode de recherche qualitative a permis aux chercheurs d'aller au-delà des résultats obtenus avec l'échelle mAIS afin d'explorer les raisons sous-jacentes d'un comportement donné et, en plus, de proposer un modèle théorique en intégrant leurs observations. Toutefois, elle montre également qu'une recherche méthodologique mixte peut être difficile à concevoir et réaliser en raison de l'expertise méthodologique nécessaire et de considérations de rigueur. Ces défis peuvent être surmontés en approfondissant ce domaine ainsi qu'en ayant recours à des collaborations de recherche interdisciplinaire.

Ce type de recherche est très important si nous souhaitons aller au-delà des questions de compétences individuelles pour offrir des soins anesthésiques sécuritaires. Étant donné que l'erreur médicale est, à l'heure actuelle, conceptualisée comme une défaillance du système, comme l'exemplifie le «modèle de fromage suisse » de Reason, ${ }^{11}$ l'étude de processus sociologiques d'envergure tels que la hiérarchie représente un autre niveau important d'analyse de conditions « latentes » ou « cachées » dans

l'environnement, lesquelles pourraient contribuer à ces erreurs. Le concept de fidélité sociologique est innovant et provocateur et pourrait faire avancer les recherches et les travaux traitant de sécurité des patients. Les méthodologies qualitatives et les méthodes mixtes sont bien adaptées à l'exploration et à la compréhension des processus et comportements sociaux. Une meilleure compréhension de ces processus pourrait entraîner une meilleure conscientisation des facteurs environnementaux qui pourront alors être abordés et enseignés de façon explicite. Ainsi, on pourra améliorer l'efficacité de la communication efficace, le fonctionnement global en équipe et, finalement, la sécurité, les soins et le devenir des patients.

Conflicts of interest None declared.

Conflit d'intérêt Aucun. 


\section{References}

1. Bould MD, Sutherland S, Sydor DT, Naik V, Friedman Z. Residents' reluctance to challenge negative hierarchy in the operating room: a qualitative study. Can J Anesth 2015; 62: this issue; DOI: 10.1007/s12630-015-0364-5.

2. Sydor DT, Bould MD, Naik VN, et al. Challenging authority during a life-threatening crisis: the effect of operating theatre hierarchy. Br J Anaesth 2013; 110: 463-71.

3. Sbaraini A, Carter SM, Evans RW, Blinkhorn A. How to do a grounded theory study: a worked example of a study of dental practices. BMC Med Res Methodol 2011; 11: 128.

4. Watling CJ, Lingard L. Grounded theory in medical education research: AMEE Guide No. 70. Med Teach 2012; 34: 850-61.

5. Bryant A, Charmaz K. Introduction. Grounded theory research; Methods and practices. In: Bryant A, Charmaz K (Eds). The Sage
Handbook of Grounded Theory. London GBR: Sage Publications; 2007: 1-28.

6. Merriam SB. Qualitative Research. A Guide to Design and Implementation. San Francisco, CA: Jossey-Bass; 2009.

7. Bryman A. Social Research Methods, 4th ed. Oxford, UK: Oxford University Press; 2012.

8. Kennedy TJ, Lingard LA. Making sense of grounded theory in medical education. Med Educ 2006; 40: 101-8.

9. Glaser BG, Strauss AL. The Discovery of Grounded Theory: Strategies for Qualitative Research. Chicago: Aldine Publications Co.; 1967.

10. Creswell JW, Plano Clark VL. Designing and Conducting Mixed Methods Research. Thousand Oaks, CA: Sage Publications; 2007.

11. Reason J. Human error: models and management. BMJ 2000; 320: 768-70. 